Vol. 1, No. 1, pp. 20-31, 2020

\title{
Calculating Premium Credibility Using the Buhlmann-Straub Model with Nonparametric Assessment
}

\author{
Dwi Susanti $^{\mathrm{a}}$, Novi Mardiana ${ }^{\mathrm{b}}$, Sukono ${ }^{\mathrm{c}}$ \\ ${ }^{\text {a,b,c }}$ Department of Mathematics, Faculty of Mathematics and Natural Sciences, Universitas Padjadjaran, Bandung, \\ INDONESIA
}

Corresponding Author: dwi.susanti@unpad.ac.id

\begin{abstract}
When an insurance company calculates the premium it will divides the policy holders into groups. The division is considered based on risk level in each group. The problem is then to devise a way of combining the experience risk of the group with the experience of the individual risk to calculate the premium, so then Credibility Theory provides a solution to this problem. This script discuss about calculation of credibility premium use BuhlmannStraub Model with nonparametric estimation to the aggregate claim amount data set within few years observation in some group of policy holders in general insurance. By using credibility theory we can calculate the value of credibility factor and credibility premium or future premium. The value of premium credibility is calculated from only one group of policyholders from the previous year's data. For better value of premium credibility, data with more experience years and the policyholder group better reflect the total loss value during the observation year. The result of this calculation are credibility factor per group, average credibility premium per members in group and credibility premium total for the last year for each group. We can obtain total losses and total premium which surprisingly equal.
\end{abstract}

Keywords: Buhlmann-Straub Model, Credibility Theory, Credibility Premium, General Insurance, Aggregate Claim Amount.

\section{Introduction}

At this time the general public began to realize the need for protection from other parties against losses or accidents that they might experience, so insurance companies took that role (Chantarat 2013; Clement, 2018). By paying premiums in each particular payment period to the guarantor in accordance with the policy that has been purchased, the insurance participants will get coverage from the insurance company when they have submitted a claim for loss / accident they experienced (Budd, 2004; Ranasinghe, 2019). 
Some people or organizations who buy insurance know that they have a tendency to make claims that are greater than the insurance company's trust in them. After providing insurance at one time, the insurance company will realize that with this, the company can increase the premiums that must be paid by the policyholders. On the other hand, policyholders believe that they deserve a lower premium. In many cases the amount of data available is too small to make a conclusion about the differences above or about the possibility of an extension of these differences. This brings us to the notion of credibility (Kim, 2013).

This paper will describe and explain the problem of premium credibility in loss insurance using the Buhlmann-Straub model with nonparametric estimates and determine the level of credibility from the previous claim data. The credibility of the premium is useful for insurance companies to determine the insurance premium and increase the accuracy of the premium seen from the experience of previous years.

\section{Materials and Methods}

\subsection{Insurance Loss}

Loss insurance is one type of insurance that provides financial coverage for all risks of loss to property or property rights of the insured. The purpose of insurance is to compensate for losses incurred, the amount of which was not predetermined (Punzo, 2018)/ In the insured loss insurance is protection only, not like life insurance that insures savings other than protection. The function of insurance itself is to divide or divert losses from uncertain events (Groot, 2019), the losses themselves are divided into three types, namely: overall losses (Total Loss), partial losses (Partial Loss), third party losses (Third Party Liability ).

\subsection{Premiums and Claims in Insurance}

Premiums are obligations that must be paid by the insured party or those who have clearly bought an insurance policy, which we then call the policyholder to the insurer in this case the insurance company. The amount of the premium is based on insurance rates where for each company the amount is different. A claim is a process by which policyholders can obtain their rights if a loss arises based on an agreement that has been agreed between the insured and the guarantor (Outreville, 1998). Claims can be paid only if the cause of the loss is recorded in the policy.

\subsection{Credibility and Premium Credibility Theories}

Credibility theory is a set of quantitative methods for managing future premiums based on past experience for insurance contracts in more or less heterogeneous portfolios. In general, statements about credibility are written as amounts the weight of the mean sample and the collective premium, the premium to be paid by the policyholder group in the portfolio.

The weight of the factor is the credibility factor. This theory was developed by combining individual risk experiences with classroom risk experiences. By using a combination of individual risk experiences, classroom risk experiences, Whitney proposes that

the premium rate is the weighted average of the following forms:

$$
X=Z \bar{X}+(1-Z) \mu
$$

The general form is written as the total weight of the sample average and the collective premium, the premium to be paid by the policyholder in the portfolio. Where, $X$ is premium credibility, $\mu$ is average total, $\bar{X}$ is average of individual risk, and $Z$ is The credibility factor is between 0 and 1 . 


\subsection{Buhlmann-Straub Model}

The Buhlmann model is the first and simplest credibility model because it effectively requires the experience of previous claims from policyholders consisting of identical and free distribution components that are associated with each previous year. For example, the insurance group will not be able to cope with the number of members insured in a group over two or more observation periods. One difficulty with this assumption is that it does not allow for variations in exposure or size. Then the Buhlmann-Straub model covers this deficiency. The main purpose of the application of credibility theory is collective insurance contracts. The credibility factor must be greater for higher risk volumes because there is no more data available.

\subsection{Non-parametric Estimates}

\subsubsection{Model for One Policy Holder}

For $i=1,2, \ldots, n+1$, for example $X_{i}$ is denoted as the amount of aggregate claims from a single policyholder during the $\mathrm{i}$ observation period. Estimated random variable value $X_{n+1}$ for period $n+1$, realized $X_{1}=x_{1}, X_{2}=x_{2}, \ldots, X_{n}=x_{n}$ for $\mathrm{n}$ periods, then this estimated value is denoted as

$$
\mathrm{E}\left[X_{n+1} \mid X_{1}=x_{1}, X_{2}=x_{2}, \ldots, X_{n}=x_{n}\right]
$$

Then the assumptions for the Buhlmann-Straub model are:

1. For $j=1,2, \ldots, n+1$, the distribution for each $X_{j}$ depends on the parameter $\Theta$.

2. For $\Theta$ given, the random variable $X_{1}, X_{2}, \ldots ., X_{n+1}$ is freely conditional and has an identical distribution.

In the Buhlmann model notated that for $i=1,2, \ldots, r$ and $j=1,2, \ldots, n$. Then

$$
\mu\left(\Theta_{i}\right)=\mathrm{E}\left[X_{i j} \mid \Theta_{i}\right]
$$

and

$$
v\left(\Theta_{i}\right)=\operatorname{Var}\left(X_{i j} \mid \Theta_{i}\right)
$$

Then it will be obtained,

$$
\mu=\mathrm{E}\left[\mu\left(\Theta_{i}\right)\right]
$$

as a whole hypothetical mean and

$$
v=\mathrm{E}\left[v\left(\Theta_{i}\right)\right]
$$

states the variance of the hypothetical mean and

$$
a=\operatorname{Var}\left(\mu\left(\Theta_{i}\right)\right)
$$

expected process variance. So by using and, respectively:

$$
\hat{\mu}=\bar{x}=\frac{1}{r} \sum_{i=1}^{r} \bar{x}_{i}=\frac{1}{r n} \sum_{i=1}^{r} \sum_{j=1}^{n} x_{i j}
$$

Where 


$$
\bar{x}_{i}=\frac{1}{n} \sum_{j=1}^{n} x_{i j}
$$

and

$$
\hat{v}=\frac{1}{r} \sum_{i=1}^{r} v_{i}=\frac{1}{r(n-1)} \sum_{i=1}^{r} \sum_{j=1}^{n}\left(x_{i j}-\bar{x}_{i}\right)^{2}
$$

where

$$
\begin{gathered}
\hat{v}_{i}=\frac{1}{n-1} \sum_{j=1}^{n}\left(x_{i j}-\bar{x}_{i}\right)^{2} \\
\mathbf{a}=\frac{1}{r-1} \sum_{i=1}^{r}\left(\bar{x}_{i}-\bar{x}\right)^{2}-\frac{v}{n} \\
=\frac{1}{r-1} \sum_{i=1}^{r}\left(\bar{x}_{i}-\bar{x}\right)^{2}-\frac{1}{r n(n-1)} \sum_{i=1}^{r} \sum_{j=1}^{n}\left(x_{i j}-\bar{x}_{i}\right)^{2}
\end{gathered}
$$

The above shows that $\hat{\mu}, \hat{v}, \hat{a}$ is an unbiased estimator of $\mu, v$ and $a$ respectively. The formula for premium credibility only depends on $\mu, v$ and $a$. No other assumptions are needed in distribution $\Theta$. Then the estimator from $\mathrm{k}$ and $\mathrm{Z}$ is obtained $\hat{k}=\frac{\hat{v}}{\hat{a}}$ and $\hat{Z}=\frac{n}{n+\hat{k}}$, where both $\hat{k}$ and $\hat{Z}$ are unbiased estimates. $k$ is a comparison between the expected value of the variance process and the variance of the conditional average. $k$ is also called the variance ratio. Equations (2.1), $\hat{k}$ and $\hat{Z}$ are estimators of Buhlmann's credibility.

So Buhlmann's estimation of $\mathrm{E}\left[X_{i, n} \mid X_{i, 1}=x_{i, 1}, X_{i, 2}=x_{i, 2}, \ldots ., X_{i, n}=x_{i, n}\right]$ is

$$
C=\hat{Z}_{i}+(1-\hat{Z}) \hat{\mu}
$$

for $i=1,2, \ldots, r$. So that the value $\hat{a}$ is not negative, the variance value should not be negative. From the Buhlmann model above, we generalize it into

$$
\mu(\Theta)=\mathrm{E}\left[X_{j} \mid \Theta\right]
$$

and

$$
v(\Theta)=m_{j} \cdot \operatorname{Var}\left(X_{j} \mid \Theta\right)
$$

for $j=1,2, \ldots, n+1$ where $m_{j}$ is a known constant that represents the number of exposures during the $\mathrm{j}$-th observation period. It is assumed that both $\mu(\Theta)$ or $v(\Theta)$ depend on the observation period. Then obtained

$$
m=\sum_{j=1}^{n} m_{j}
$$

the difference between this model and the classical model of Buhlmann is that in this model $X_{j}$ represents the average number of claims per unit of exposure rather than the aggregate number of claims.

Theorem 3.1 Let $X_{1}, X_{2, \ldots}$ be random variables with a mean $\Theta$ such that 


$$
E\left[X_{i} \mid \Theta\right]=\mu(\Theta)
$$

where $i=1,2 \ldots$ and $X_{i}$ are free to conditional on $\Theta$. Then for $n=1,2 \ldots$

$$
\begin{gathered}
E\left[X_{n+1} \mid X_{1}=x_{1}, X_{2}=x_{2}, \ldots, X_{n}=x_{n}\right] \\
=E_{\Theta}\left[\mu(\Theta)\left|X_{n+1}\right| X_{1}=x_{1}, X_{2}=x_{2}, \ldots ., X_{n}=x_{n}\right]
\end{gathered}
$$

As in the Buhlmann model a linear estimate is chosen from $\mu(\Theta)$ conditional expectations from the form

$$
a_{0}+a_{1} x_{1}+a_{2} x_{2}+\ldots+a_{n} x_{n}
$$

The linear estimation chosen is one that has a coefficient that is the quantity of $a_{0}, a_{1}, a_{2} \ldots a_{n}$, by minimizing

$$
\mathrm{E}\left[\left(\mu(\Theta)-a_{0}-a_{1} x_{1}-a_{2} x_{2}-\ldots-a_{n} x_{n}\right)^{2}\right]
$$

The coefficient of $a_{0}, a_{1}, a_{2} \ldots a_{n}$ is obtained by deriving the equation (2.20) $n+l$ times. First with respect to $a_{0}$ then with respect to $a_{1}$ and so on up to $a_{n}$ and equate each child equal to zero. Generate $\mathrm{n}+1$ equations to solve $a_{0}, a_{1}, a_{2} \ldots a_{n}$. The result is

$$
a_{0}, a_{1}, a_{2} \ldots a_{n}
$$

where the $\mathrm{Z}$ value is:

$$
Z=\frac{\sum m_{j}}{\sum m_{j}+\mathrm{E}[v(\Theta)] / \operatorname{Var}(\mu(\Theta))}=\frac{m}{m+v / a}
$$

where $m$ is the total exposure associated with the policyholder and $Z$ Buhlmann-Straub credibility or credibility factors that depend on $m$, and

$$
a_{j}=\frac{m_{j}}{\sum m_{j}+\mathrm{E}[v(\Theta)] / \operatorname{Var}(\mu(\Theta))}
$$

for $j=1,2, \ldots, n$. Then the point estimator of conditional expectations $\mu(\Theta)$ is

$$
Z . \bar{X}+(1-Z) \cdot \mathrm{E}[\mu(\Theta)]
$$

where

$$
\bar{X}=\frac{\sum m_{j} X_{j}}{\sum m_{j}}
$$

It can be seen that the equation above is a linear function of the random variable $X_{1}, X_{2}, \ldots . X_{n}$ where $\bar{X}$ is a linear function of $X_{1}, X_{2}, \ldots . X_{n} . \bar{X}$ is the average weighting of $X_{j}$, with weights proportional to $m_{j}$. Following group interpretation, $X_{j}$ is the average loss from group members $m_{j}$ in year $j$ and then $m_{j} X_{j}$ is the total loss from the group in year $j$. Then $\bar{X}$ is the overall average loss per group member for $n$ years. The premium credibility that must be charged to the group in year $n+1$ will be $m_{n+1}[Z \bar{X}+(1-Z) \mu]$ for $m_{n+1}$ members the 
following year.

\subsubsection{Model for Two or More Policy Holders}

For example $r(r \geq 2)$ the group of policyholders where the $\mathrm{i}^{\text {th }}$ policyholder has $n_{i}$ data years for $i=1,2, \ldots, r$. For example $m_{i j}$ states the number of exposure units for the $\mathrm{i}^{\text {th }}$ policyholder during the $\mathrm{j}^{\text {th }}$ policy year, $j=1,2, \ldots, n+1$. For example $X_{i j}$ represents a random variable that represents the average number of claims per unit of exposure of the $\mathrm{i}^{\text {th }}$ policyholder during the $\mathrm{j}$-policy year.

Let $X_{i}=\left(X_{i, 1}, X_{i, 2}, \ldots, X_{i, n}\right)$ be a random vector of the average number of claims for the $\mathrm{i}-\mathrm{i}$ policyholder, $i=1,2, \ldots, r$. Then the assumptions that must exist are:

1) Random vectors $X_{1}, X_{2}, \ldots, X_{n}$ are assumed to be independent of each other. This means that the experience of one policyholder does not affect different policyholders.

2) For $i=1,2, \ldots, r$, the distribution of each element of $X_{i}$ depends on the risk parameter $\theta_{i}$ (unknown), where $\theta_{i}$ is the realization of the random variable $\Theta_{i}$.

3) Random variables $\Theta_{1}, \Theta_{2}, \ldots, \Theta_{r}$ are independent and have identical distributions

4) For i and $\Theta_{i}$ the random variable $X_{i, 1}, X_{i, 2}, \ldots ., X_{i, n}$ is conditionally independent of each other.

These assumptions are similar to the Buhlmann model. Here the generalization is done simply by defining that,

$$
\begin{gathered}
\mu\left(\Theta_{i}\right)=\mathrm{E}\left[X_{i j} \mid \Theta_{i}\right] \\
\operatorname{Var}\left(\Theta_{i}\right)=m_{i j} \operatorname{Var}\left(X_{i j} \mid \Theta_{i}\right)
\end{gathered}
$$

for $i=1,2, \ldots, r$ and $j=1,2, \ldots, n_{i}$.

The total number of exposure units caused by the $n_{i}^{\text {th }}$ policyholder during the first policy year is:

$$
m_{i}=\sum_{j=1}^{n_{i}} m_{i j}
$$

and all the sum of the exposure units is

$$
m=\sum_{i=1}^{r} m_{i}
$$

$m_{i j}=1$ for all $\mathrm{i}$ and $\mathrm{j}$. The assumptions used in this model are less restrictive than the classic Buhlmann model.

Then the estimator of the hypothetical mean $(\mu)$, expected process variance $(v)$ and the variance of the hypothetical mean $(a)$ are as follows:

$$
\begin{gathered}
\hat{\mu}=\bar{X}=\frac{1}{m} \sum_{i=1}^{r} m_{i} \bar{X}_{i}=\frac{1}{m} \sum_{i=1}^{r} \sum_{j=1}^{n_{i}} m_{i j} X_{i j} \\
\bar{X}=\frac{1}{m_{i}} \sum_{j=1}^{n_{i}} m_{i j} X_{i j} \\
\hat{v}=\frac{\sum_{i=1}^{r} \sum_{j=1}^{n_{i}} m_{i j}\left(X_{i j}-\bar{X}_{i}\right)^{2}}{\sum_{i=1}^{r}\left(n_{i}-1\right)}
\end{gathered}
$$




$$
\hat{a}=\left\{m-\frac{1}{m} \sum_{i=1}^{r} m_{i}^{2}\right\}^{-1}\left\{\sum_{i=1}^{r} m_{i}\left(\bar{X}_{i}-\bar{X}\right)^{2}-\hat{v}(r-1)\right\}
$$

where $\hat{v}$ has been defined in equation (32).

It can be seen that $\hat{\mu}, \hat{v}$ and $\hat{a}$ are all unbiased estimates of $\mu, v$ and $a$. All three are nonparametric estimates without distribution assumptions. These three estimators are not the only commonly used unbiased estimators and the value $\hat{a}$ can be negative. There is a possibility that the value $\hat{a}$ is negative if the variance is negative. Estimates from $k$ is $\hat{k}=\frac{\hat{v}}{\hat{a}}$ and estimators of the credibility factor for $\mathrm{i}$ policyholders, $Z_{i}$ is

$$
\hat{Z}_{i}=\frac{m_{i}}{m_{i}+\hat{k}}
$$

Then the premium credibility estimator for the following year per unit of exposure according to the Buhlmann-Straub model is

$$
\hat{X}_{i}=\hat{Z}_{i} \bar{X}_{i}+\left(1-\hat{Z}_{i}\right) \mu
$$

for $i=1,2, \ldots, r$. Although $\hat{v}$ and $\hat{a}$ are unbiased estimators of $v$ and $a$, this assumption does not apply to $\hat{k}$ and $\hat{Z}_{i}$. And the Buhlmann-Straub compromise estimator of the aggregate amount of the claim is for the I policyholder next year where for $i=1,2, \ldots, r$ is:

$$
\hat{C}_{i}=m_{i 3} \cdot \hat{X}_{i}
$$

There is one thing that must be considered if the credibility value is sought using the formula above. In the past, data from the $\mathrm{j}^{\text {th }}$ policyholder was collected in the form of exposure $m_{i}$. The total loss to all policyholders was

$$
T L=\sum_{i=1}^{r} m_{i} \bar{X}_{i}
$$

If the credibility of the premium is charged as above, the total premium will be:

$$
\begin{aligned}
T P & =\sum_{i=1}^{r} m_{i}\left[\hat{Z}_{i} \bar{X}_{i}+\left(1-\hat{Z}_{i}\right) \hat{\mu}\right] \\
& =\sum_{i=1}^{r} m_{i}\left(1-\hat{Z}_{i}\right)\left(\hat{\mu}-\bar{X}_{i}\right)+\sum_{i=1}^{r} m_{i} \bar{X}_{i} \\
& =\sum_{i=1}^{r} m_{i} \frac{\hat{k}}{m_{i}+\hat{k}}\left(\hat{\mu}-\bar{X}_{i}\right)+\sum_{i=1}^{r} m_{i} \bar{X}_{i}
\end{aligned}
$$

TP and TL values are expected to be the same because if the premium increases and will meet a policy based on the level of total claims from previous experience. Then we need to assume again that:

$$
0=\sum_{i=1}^{r} m_{i} \frac{\hat{k}}{m_{i}+\hat{k}}\left(\hat{\mu}-\bar{X}_{i}\right)
$$




$$
\begin{gathered}
\hat{\mu} \sum_{i=1}^{r} \hat{Z}_{i}=\sum_{i=1}^{r} \hat{Z}_{i} \bar{X}_{i} \\
\hat{\mu}=\frac{\sum_{i=1}^{r} \hat{Z}_{i} \bar{X}_{i}}{\sum_{i=1}^{r} \hat{Z}_{i}}
\end{gathered}
$$

Estimating the value in this way has an advantage compared to equation (2.41), which is preserving total claims. The thing to remember is that when equation (2.33) is used it must also use equation (2.31). The weight in equation (2.40) results in the smallest unconditional variance of $\hat{\mu}$. This means the value of premium credibility with $\hat{\mu}$ alternative will be better. Total past premium credibility is equivalent to current total loss.

\subsection{Iteration Process in the Buhlmann-Straub Model}

The steps that must be taken to get the value of premium credibility and credibility factors are:

Step 1: Determine the number of policyholders, $r \geq 2$.

Step 2: Determine the period of observation $n_{i}$, for each policy holder $r$.

Step 3: Determine exposure measurements, $m_{i j}$, for each policyholder during each observation period.

Step 4: Calculate the average number of claims, $X_{i j}$, for each policyholder during each observation period.

Step 5: Calculate the average number of claims, $\bar{X}_{i}$, during the entire observation period for each policyholder.

Step 6: Calculate the estimated overall mean, $\hat{\mu}$.

Step 7: Calculate the estimated process variance estimation, $\hat{v}$.

Step 8: Calculate the estimated variance from the conditional average, $\hat{a}$.

Step 9: Calculate the value $\hat{k}=\frac{\hat{v}}{\hat{a}}$.

Step 10: Calculate the credibility factor, $\hat{Z}_{i}$, for each policyholder.

Step 11: Calculate the estimated Buhlmann-Straub compromise, $\hat{X}_{i}$ from the average number of claims per unit of exposure for each policyholder.

Step 12: Calculate the estimated Buhlmann-Straub compromise, $\hat{C}_{i}$ of the aggregate claims for each policyholder.

Step 13: Calculate TP and TL values.

Step 14: Calculate the new value $\hat{\mu}$.

Step 15: Calculate the value of new premium credibility.

\section{Results and Discussion}

Table 1 and Table 2 below show the data used in the study. After calculating based on the Buhlmann Straub Model discussed earlier, the calculation results are presented in Table 3, Table 4, table 5 and Table 6. 
Table 1 Aggregate Claim Data for One Group

\begin{tabular}{ccccc}
\hline Policy Holder & & \multicolumn{3}{c}{ Policy Year } \\
\cline { 3 - 5 } Group & & 1 & 2 & 3 \\
\hline 1 & Large aggregate claims & 60000 & 70000 & - \\
& Size in groups & 125 & 150 & 200 \\
\hline
\end{tabular}

Table 2 Aggregate Data Claims of Five Groups

\begin{tabular}{cccccc}
\hline \multirow{2}{*}{$\begin{array}{c}\text { Policy Holder } \\
\text { Group }\end{array}$} & & \multicolumn{4}{c}{ Policy Year } \\
\cline { 3 - 6 } & & 1 & 2 & 3 & 4 \\
\hline 1 & Total aggregate claims & - & 20000 & 25000 & - \\
& Size in groups & - & 100 & 120 & 110 \\
2 & Large aggregate claims & 19000 & 18000 & 17000 & - \\
& Size in groups & 90 & 75 & 70 & 60 \\
3 & Large aggregate claims & 26000 & 30000 & 35000 & - \\
& Size in groups & 150 & 175 & 180 & 200 \\
4 & Large aggregate claims & 8000 & 11000 & 15000 & - \\
& Size in groups & 40 & 50 & 75 & 75 \\
& Large aggregate claims & 20000 & 24000 & 18000 & - \\
& Size in groups & 100 & 120 & 120 & 95 \\
\hline
\end{tabular}

Table 3 Summary of Calculation Results for One Group

\begin{tabular}{cccccccc}
\hline Grup & $\bar{X}_{i}$ & $\hat{v}_{i}$ & $\hat{a}_{i}$ & $\hat{k}$ & $\hat{Z}$ & $\hat{X}_{i}$ & $\hat{C}_{i}$ \\
\hline 1 & 472.73 & 12.115 .15 & 699.60 & 17.32 & 0.94 & 474.37 & 94.874
\end{tabular}

After an iterative procedure, the final estimate of $\bar{X}_{i}, \hat{v}_{i}, \hat{a}, \hat{k}, \hat{Z}, \hat{X}_{i}, \hat{C}_{i}$ for data with a group of policyholders is obtained as shown in Table 3 above.

Analysis of the general results, namely:

Calculations with the amount of data like this are not recommended, because the conclusions and the results obtained will be less accurate, unless there are no other alternatives. For better results, the calculated data should be more in terms of the number of policy-holder groups and the number of years observed.

Here is a table of calculations for data with five groups for four years. 
Table 4 Exposure Size for each experience year

\begin{tabular}{|c|c|c|c|}
\hline \multicolumn{2}{|c|}{ Exposure Size } & \multirow{2}{*}{$\frac{m_{33}}{m_{34}}$} & \multirow{2}{*}{$\frac{150}{200}$} \\
\hline$m_{11}$ & 100 & & \\
\hline$m_{12}$ & 120 & $m_{41}$ & 40 \\
\hline$m_{13}$ & 110 & $m_{42}$ & 50 \\
\hline$m_{21}$ & 90 & $m_{43}$ & 75 \\
\hline$m_{22}$ & 75 & $m_{44}$ & 75 \\
\hline$m_{23}$ & 70 & $m_{51}$ & 100 \\
\hline$m_{24}$ & 60 & $m_{52}$ & 120 \\
\hline$m_{31}$ & 150 & $m_{53}$ & 120 \\
\hline$m_{32}$ & 175 & $m_{54}$ & 95 \\
\hline
\end{tabular}

Table 5 Overall Calculations of Five Policy Holder Groups

\begin{tabular}{cccccccccc}
\hline Group & $m_{i}$ & $\bar{X}_{i}$ & $\hat{\mu}$ & $\hat{v}$ & $\hat{a}$ & $\hat{k}$ & $\hat{Z}_{i}$ & $\hat{X}_{i}$ & $\hat{C}_{i}$ \\
\hline 1 & 220 & 204.54 & & & & & 0.73 & 202.02 & 22222.2 \\
2 & 235 & 229.79 & & & & & 0.74 & 220.80 & 13248 \\
3 & 505 & 180.19 & 195.22 & 35.565 .85 & 436.22 & 81.53 & 0.86 & 182.29 & 36458 \\
4 & 165 & 206.06 & & & & & 0.67 & 202.48 & 15186 \\
5 & 340 & 182.35 & & & & & 0.80 & 184.92 & 17567.4 \\
\hline
\end{tabular}

The value of Total Losses is obtained through equation (2.37). Then the value of Total Losses for the data above are:

$T L=220(204,54)+235(229,79)+505(180,19)+165(206,06)+340(182,35)$

\section{$=285.994,3$}

While the value of Total Premium is calculated using equation (2.38) by entering the required value from existing data. Then the results of the calculation of the total premiums are:

$$
\begin{gathered}
T P=\sum_{i=1}^{r} m_{i}\left[\hat{Z}_{i} \bar{X}_{i}+\left(1-\hat{Z}_{i}\right) \hat{\mu}\right] \\
=\sum_{i=1}^{r} m_{i}\left(1-\hat{Z}_{i}\right)\left(\hat{\mu}-\bar{X}_{i}\right)+\sum_{i=1}^{r} m_{i} \bar{X}_{i} \\
=\sum_{i=1}^{r} m_{i} \frac{\hat{k}}{m_{i}+\hat{k}}\left(\hat{\mu}-\bar{X}_{i}\right)+\sum_{i=1}^{r} m_{i} \bar{X}_{i}=285.998,80
\end{gathered}
$$

TL value is equal to TP. This happens because if there is an increase in premiums approved by the regulator then it is usually based on the level of total claims from previous experience. Without seeing any errors, the TP value corresponds to the actual loss of 286000 . .

In order for the credibility of the premium value to be more accurate, new values $\hat{\mu}$ must be sought with equation (2.41). Then by entering the required values, the new estimated value $\hat{\mu}$ is obtained 199,54.. So we get 
an estimate of the new premium credibility as shown in the following table:

Table 6 New Premium Credibility

\begin{tabular}{cccc}
\hline$\hat{X}_{i}$ & $\begin{array}{c}\text { Estimated credibility of the New } \\
\text { Premium }\end{array}$ & $\hat{C}_{i}$ & New Estimated Total Credibility \\
\hline 1 & 203.19 & 1 & 22350.9 \\
2 & 221.93 & 2 & 13315.8 \\
3 & 182.89 & 3 & 36578 \\
4 & 203.90 & 4 & 15292.5 \\
5 & 185.78 & 5 & 17649.1 \\
\hline
\end{tabular}

The following table presents a comparison of the value of new total premium credibility with the original:

Table 7 Comparison of Initial and Improved Credibility Estimates

\begin{tabular}{ccc}
$\begin{array}{c}\text { Estimated Premium } \\
\text { Credibility }\end{array}$ & $\begin{array}{c}\text { Estimated premium } \\
\text { credibility improved }\end{array}$ & $\begin{array}{c}\text { Percentage of } \\
\text { Increase }\end{array}$ \\
\hline 22350.9 & 22222.2 & $0.57 \%$ \\
13315.8 & 13248 & $0.51 \%$ \\
36578 & 36458 & $0.33 \%$ \\
15292.5 & 15186 & $0.70 \%$ \\
17649.1 & 17567.4 & $0.46 \%$ \\
\hline
\end{tabular}

In the second data with the number of 5 policyholder groups and 4 years of observation, indeed there is an increase in the value of premium credibility, both for the average member in one policyholder group and the total for each group of policyholders, if the new $\hat{\mu}$ value is used for get a new premium credibility value. The credibility of the average and total premium produced is more accurate. There is still an increase even though the credibility factor used and the values of other variables have not changed. The value of new premium credibility is greater than the value previously estimated. This means that the value is getting closer to the future premiums that must be paid by policyholders in each group. This credibility value states the amount of premium to be paid per group of policyholders. Here the values generated are free from each other. There is no influence between the group policyholders.

\section{Conclussion}

The value of premium credibility can be calculated only from one group of policyholders from the previous year's data. For better value of premium credibility, data with more experience years and group policyholders will better reflect the total loss value during the observation year. Application of the Buhlmann-Straub model the available data results in a credibility factor $Z$ from each group so that the premium credibility value for each group can be generated either on average or in total. Premium credibility value per group and exposure size of each group affect the value of total premium credibility for the year 4 The greater the credibility factor means the value $Z \rightarrow 1$ then the credibility of the individual premium is stronger than the credibility of the collective $\backslash$ group premium and if $Z=0$ there is no heterogeneity in the portfolio which means the group's mean is the best estimator for credibility. 


\section{References}

Budd, J., McCall, B., 2004. Unions and unemployment insurance benefits receipt: Evidence from the current population survey. Ind. Relations 43, 339-355.

Chantarat, S., Mude,A.G., Barrett,C.B., Carter,M.R. (2013) Designing index-based livestock insurance for managing asset risk in Northern Kenya. The Journal of Risk and Insurance 80 (1): 205-237.

Clement, K. Y., Wouter Botzen, W. J., Brouwer, R., \& Aerts, J. C. J. H. (2018). A global review of the impact of basis risk on the functioning of and demand for index insurance. International Journal of Disaster Risk Reduction, 28, 845-853. doi:10.1016/j.ijdrr.2018.01.001

Goda, K., \& Ren, J. (2013). Seismic risk management of insurance losses using extreme value theory and copula. Handbook of Seismic Risk Analysis and Management of Civil Infrastructure Systems, 760 786. doi:10.1533/9780857098986.5.760

Goovaerts, M.J., Kaas R., Van Heerwaarden, A.E. \& Bauwelinckx,T. 1990 . Effective Actuarial Methods. Amsterdam : Elsevier Science Ltd.

Groot, N. Y., \& Klaauw B. V. D. (2019). The effects of reducing the entitlement period to unemployment insurance benefits. Labour Econimics, 57, 195-208. https://doi.org/10.1016/j.labeco.2019.02.003

Hao, M., Macdonald, A. S., Tapadar, P., \& Thomas, R. G. (2016) Insurance loss coverage under restricted risk classification: The case of iso-elastic demand. ASTIN Bulletin.

Hao, M., Macdonald, A. S., Tapadar, P., \& Thomas, R. G. (2018). Insurance loss coverage and demand elasticities. Insurance: Mathematics and Economics, 79, 15-25.

Kass, R., Goovaerts,.M., Dhaene, Jan., \& Kluwer, M. D. 2000. Modern Actuarial Risk Theory. Boston : Academic Publisher.

Kim, J. H. T., \& Jeon, Y. (2013). Credibility theory based on trimming. Insurance: Mathematics and Economics, 53(1), 36-47. doi:10.1016/j.insmatheco.2013.03.012

Klugman,S.A., Panjer,H.,.Harry., \& Willmot, E.Gordon .1998. Loss Models: Data to Decisions. New York : A Wiley Interscience Publication.

Punzo, A., Bagnato, L., \& Maruotti, A. (2018). Compound unimodal distributions for insurance losses. Insurance: Mathematics and Economics, 81, 95-107. doi:10.1016/j.insmatheco.2017.10.007

Outreville, J. (1998). Insurance Concepts. Theory and Practice of Insurance, pp. 131-146, springer ISBN: 978-1-4615-6187-3. DOI: 10.1007/978-1-4615-6187-3. 